\title{
MARCADORES DE INFECCIÓN PARA HEPATITIS VIRAL EN DONANTES DE SANGRE DE UN HOSPITAL NACIONAL DE LIMA METROPOLITANA
}

\author{
Juan Morales ${ }^{1, a}$, José Fuentes-Rivera2,a, Carlos Delgado-Silva ${ }^{3, a}$, Hernán Matta-Solís ${ }^{1, a}$
}

\begin{abstract}
RESUMEN
El objetivo del estudio fue determinar la frecuencia de marcadores de infección para hepatitis $B$, hepatitis $C$ y conocer los factores asociados en los donantes de sangre. El estudio se realizó con datos del registro de donantes de un hospital público de Lima. De 28263 sujetos analizados entre 2012 y 2015, el 0,6\% $(n=156)$ fue reactivo para HBsAg; $5,2 \%$ $(n=1465)$ para anti-HBc, y $0,8 \%(n=232)$ para Anti-HVC. Los resultados positivos para HBsAg $(p=0,319)$ y anti-HVC $(p=0,037)$ fueron en mayor proporción en los donantes voluntarios. Los resultados positivos para HBsAg y anti-HBc fueron en mayor proporción en las personas de 50 a más años de edad. Los donantes voluntarios fueron en mayor proporción en los sujetos menores de 20 años $(p<0,001)$. En conclusión, la reactividad a los marcadores de infección para hepatitis está asociado a la donación voluntaria y al grupo de edad de los donantes.
\end{abstract}

Palabras clave: Hepatitis B; Hepatitis C; Donantes de sangre (Fuente: DeCS, BIREME).

\section{VIRAL HEPATITIS INFECTION MARKERS AMONG BLOOD DONOR IN A NATIONAL HOSPITAL OF METROPOLITAN LIMA}

\begin{abstract}
The aim of the study was to determine the frequency of infection markers for hepatitis $B$ and hepatitis $C$ and to identify associated factors in blood donors. The study was carried out using data obtained from blood donor medical records collected in a public hospital in Lima. Of 28,263 individuals analyzed between 2012 and 2015, 0.6\% $(n=156)$ were reactive for HBsAg; 5.2\% ( $n=1,465)$, for anti-HBc; and 0.8\% ( $n=232)$, for anti-HVC. Positive results for HBsAg $(p=0.319)$ and anti-HVC $(p=0.037)$ were more common in voluntary donors. For HBsAg and anti-HBc, positive results were more common in individuals aged 50 years or older. The number of voluntary donors was higher among individuals younger than 20 years $(p<0.001)$. The study indicates that reactivity to hepatitis infection markers is associated with voluntary donation and the age group of blood donors.
\end{abstract}

Key words: Hepatitis B; Hepatitis C; Blood donors (Source: MeSH, NLM).

\section{INTRODUCCIÓN}

La hepatitis viral, una pandemia silenciosa que genera una enorme carga para los sistemas de salud, es la octava causa de mortalidad en todo el mundo. Se estima que cada año ocasiona 1,4 millones de muertes por infección aguda, cáncer hepático y cirrosis relacionadas con la hepatitis, de los cuales el $55 \%$ son atribuidos al virus de la hepatitis $\mathrm{B}$ (VHB) y el $35 \%$ al virus de la hepatitis $\mathrm{C}(\mathrm{VHC})^{(1)}$. A nivel mundial, se estima que 2000 millones de personas están afectadas por el VHB, de ellas, 240 millones padecen infección hepática crónica por el VHB y corren el riesgo de fallecer por cirrosis o carcinoma hepatocelular, enfermedades que, se calcula, provocan de 500000 a 700000 muertes por año en el mundo (2).

La prevalencia del VHB es variable según las regiones, se estima de 5 a $8 \%$ en África y de 2 a $4 \%$ en Sudamérica (2); la prevalencia reportada de HBsAg fue de $2 \%$ en los países de la Región Este del Mediterráneo y Medio Oriente ${ }^{(3)}$. El Perú es considerado entre los países de endemicidad

\footnotetext{
Universidad de Ciencias y Humanidades. Lima, Perú.

Universidad Nacional Mayor de San Marco. Lima, Perú.

Hospital Nacional Dos de Mayo. Lima, Perú.

Médico cirujano

Recibido: 15/11/2016 Aprobado: 09/08/2017 En línea: 29/09/2017
}

Citar como: Morales J, Fuentes-Rivera J, Delgado-Silva C, Matta-Solís H. Marcadores de infección para hepatitis viral en donantes de sangre de un hospital nacional de Lima metropolitana. Rev Peru Med Exp Salud Publica. 2017;34(3):466-71.doi: 10.17843/rpmesp.2017.343.2503 
intermedia para $\mathrm{VHB}$, sin embargo, varía según las regiones, en la costa es baja ( $\mathrm{HBsAg}<1 \%$ ), en la selva la endemicidad se ubica entre mediana (HBsAg 2 a $7 \%$ ) y alta endemicidad (HBsAg >8\%), llegando hasta $83 \%$ en la población indígena ${ }^{(4)}$. Mayca y Vallejos reportaron presencia del HBsAg en la costa $(0-4,8 \%)$, sierra $(13,8-$ $16 \%)$ y selva $(3-17,1 \%){ }^{(5)}$. Según el Centro Nacional de Epidemiología, Prevención y Control de Enfermedades, en los últimos 7 años los casos de hepatitis B han mostrado una tendencia ascendente, de 291 casos en 2009 a 1174 casos en 2015, siendo Lima, Ayacucho, Arequipa, Cusco y Loreto los departamentos que más casos han reportado.

Existen distintas formas de transmisión de los virus de la VHB y VHC, siendo una vía potencial a través de la transfusión sanguínea y, más aún, considerando la endemicidad variable en nuestro país, los bancos de sangre de los hospitales hacen el tamizaje para detectar la infección por VHB y VHC. Por otra parte, la Organización Mundial de la Salud (OMS) sostiene que la sangre procedente de donantes voluntarios altruistas es más segura que la sangre de donantes de reposición que proceden de los familiares o conocidos; sin embargo, en los bancos de sangre del país el $95 \%$ del abastecimiento de sangre procede de donantes de reposición y el $86 \%$ de la sangre recolectada en el Perú se obtienen en Lima ${ }^{(6)}$. EI presente trabajo tiene como objetivos estimar la frecuencia de marcadores de infección para hepatitis $B$, hepatitis $C$ y conocer los factores asociados en los donantes que acuden al servicio de Banco de Sangre de un hospital nacional de Lima Metropolitana.

\section{EL ESTUDIO}

\section{DISEÑO DEL ESTUDIO}

Estudio observacional transversal retrospectivo, desarrollado en el Banco de Sangre del Hospital Nacional Dos de Mayo en el periodo 2012 a 2015.

\section{POBLACIÓN Y MUESTRA}

De un total de 50127 sujetos registrados en el periodo 2012 al 2015 , el $43,6 \%(n=21864)$ fueron excluidos en la fase de selección y la entrevista, al 56,4\% ( $n=28$ 263) restante se les realizó la prueba de tamizaje inmunoserológico.

Basados en el protocolo institucional, los postulantes para la donación de sangre deben pasar por el proceso de selección del donante y la entrevista. En la fase de selección se realiza la evaluación del peso, la accesibilidad de las venas y el nivel de hemoglobina; los sujetos aptos son registrados en el sistema informático y continúan con la entrevista donde se realiza la identificación de los riesgos, la autoexclusión voluntaria

\section{MENSAJES CLAVE}

Motivación para realizar el estudio. En el Perú, el 95\% del abastecimiento de sangre procede de donantes de reposición, ya sea por los familiares o conocidos y, según los organismos internacionales de salud, es menos segura que los donantes voluntarios.

Principales hallazgos. La presencia de hepatitis B y hepatitis C fue más frecuente en los donantes voluntarios.

Implicancias. La donación periódica o repetitiva en los donantes de reposición y en los voluntarios puede contribuir a obtener sangre segura, por tanto, tienen menos riesgo de transmisión de hepatitis $\mathrm{B}$ y hepatitis $\mathrm{C}$.

y el consentimiento informado. Los sujetos que son admitidos en la entrevista, se someten al tamizaje inmunoserológico para la detección de los principales agentes infecciosos considerados obligatorios según la normativa nacional, entre ellos los exámenes para el VHB y VHC, y en función de los resultados son calificados como donantes aptos o son excluidos.

Los criterios de inclusión considerados en el presente estudio fueron todos los sujetos con tamizaje inmunoserológico registrados en el sistema informático con los términos reactivos $(R)$ y no reactivos (NR), excluyéndose los resultados indeterminados o los que no tenían registro.

\section{VARIABLES DE ESTUDIO}

Inmunoserología para VHB: determinado por la presencia de los antígenos de superficie del VHB (HBsAg) y anticuerpos del core (anti-HBc) en la prueba de tamizaje. Inmunoserología para VHC: determinado por la presencia de anticuerpos frente al virus de la hepatitis C (anti-VHC)

El método empleado para la determinación de los marcadores fue la quimioluminiscencia (CLIA) utilizando el kit del laboratorio ABBOTT ${ }^{\circledR}$.

\section{ANÁLISIS ESTADÍSTICO}

Los datos del banco de sangre fueron exportados a Microsoft Excel y se procedió con el ordenamiento de los datos. Las principales variables del estudio fueron organizadas en tablas de frecuencias y se determinaron los principales estadísticos descriptivos. La frecuencia de positividad de los marcadores $\mathrm{HBsAg}$, anti-HBc y antiHVC se realizó a partir de la proporción en porcentaje de los resultados $R$ respecto a la suma de $R$ y NR [R/ $(\mathrm{R}+\mathrm{NR})$ ] para cada marcador. Los criterios del donante 
voluntario y no voluntario se recogieron del registro informático del banco de sangre. Para determinar la asociación de las variables se empleó el estadístico chi cuadrado $\left(X^{2}\right)$, considerándose valores de $p<0,05$ como significativo. Los programas empleados fueron el Microsoft Excel 2010 y el IBM SPSS Statistics 23.

\section{ASPECTOS ÉTICOS}

El proyecto fue ejecutado con el consentimiento de los responsables del Banco de Sangre y aprobado por el Comité de Ética de la Universidad de Ciencias y Humanidades (Acta CEI 002-2016).

\section{HALLAZGOS}

Participaron sujetos con edades entre 17 y 70 años, con una media de 33,49 (DE 10,01), la mayoría fueron varones entre los 20 y 39 años. La proporción de donantes voluntarios fue menor al $5 \%$, descendiendo hasta el $1 \%$ en el 2015 (Tabla 1 ).

Con relación a los marcadores, la proporción de reactividad para $\mathrm{HBsAg}$ mantiene una tendencia creciente desde $0,3 \%$ en 2012 hasta $0,7 \%$ en 2015; anti-HBc de 2,1\% en 2012 hasta 7,3 \% en 2015; VHC de $0,7 \%$ en el 2012 hasta $1,0 \%$ en 2014 , descendiendo a $0,9 \%$ en 2015. En el acumulado de 2012 a 2015, de los 28263 sujetos con tamizaje inmunoserológico, el $0,6 \%(n=156)$ fue reactivo para $\mathrm{HBsAg} ; 5,2 \%(n=1465)$ reactivo para anti-HBc y el $0,8 \%(n=232)$ reactivo para anti-VHC (Tabla 2).
Según la categoría de los postulantes, en el grupo voluntario los resultados reactivos para HBsAg fue en mayor proporción, mientras que para anti-HBc fueron en el grupo no voluntario; en ambos casos, la diferencia no fue significativa $(p>0,05)$. Los resultados reactivos para antiVHC fueron en mayor proporción en el grupo voluntario, con diferencias significativas $(p=0,037)$ (Tabla 3$)$.

Considerando el grupo de edad, los resultados positivos para el HBsAg fueron en menor proporción en los menores de 20 años y entre los 30 y 50 años. Los resultados positivos tanto para el $\mathrm{HBsAg}$ como para el anti-HBc se presentaron en mayor proporción en los sujetos de 50 a más años de edad, con diferencias significativas $(p<0,001)$ para el marcador anti-HBc. Los casos positivos para el anti-VHC se presentaron en mayor proporción en los menores de 20 años $(p=0,174)$. Los donantes voluntarios fueron en mayor proporción en los sujetos menores de 20 años ( $p<0,001)$ (Tabla 4).

\section{DISCUSIÓN}

En el proceso de selección de los donantes de sangre, en nuestro estudio la mayoría de los participantes fueron varones. Existen factores biológicos o condición médica que pueden limitar la donación en las mujeres, tales como el peso, edad y la intensidad de la menstruación, que están vinculadas a la deficiencia de hierro ${ }^{(7)}$, los cuales pueden ser motivos de exclusión de las mujeres en la fase de selección del donante.

En comparación con otros hospitales públicos de Lima, la proporción de resultados positivos para $\mathrm{HBsAg}$

Tabla 1. Características generales de los donantes de sangre en el Hospital Nacional Dos de Mayo, Lima 2012-2015

\begin{tabular}{|c|c|c|c|c|c|c|c|c|c|c|}
\hline \multirow{2}{*}{ Características } & \multicolumn{2}{|c|}{2012} & \multicolumn{2}{|c|}{2013} & \multicolumn{2}{|c|}{2014} & \multicolumn{2}{|c|}{2015} & \multicolumn{2}{|c|}{ Total } \\
\hline & $\mathbf{n}$ & $\%$ & $\mathbf{n}$ & $\%$ & $\mathbf{n}$ & $\%$ & $\mathbf{n}$ & $\%$ & $\mathbf{n}$ & $\%$ \\
\hline \multicolumn{11}{|l|}{ Sexo } \\
\hline Femenino & 3621 & 33,4 & 3950 & 32,7 & 3273 & 30,4 & 4294 & 26,1 & 15138 & 30,2 \\
\hline Masculino & 7030 & 64,9 & 8131 & 67,3 & 7485 & 69,6 & 12148 & 73,9 & 34794 & 69,4 \\
\hline Sin datos & 185 & 1,7 & 0 & 0,0 & 2 & 0,0 & 8 & 0,1 & 195 & 0,4 \\
\hline \multicolumn{11}{|l|}{ Grupo de edad (años) } \\
\hline$<20$ & 887 & 8,2 & 1005 & 8,3 & 976 & 9,1 & 1758 & 10,7 & 4626 & 9,2 \\
\hline $20-29$ & 3309 & 30,5 & 3668 & 30,4 & 3336 & 31 & 5126 & 31,2 & 15439 & 30,8 \\
\hline $30-39$ & 3362 & 31 & 3587 & 29,7 & 3206 & 29,8 & 4703 & 28,6 & 14858 & 29,6 \\
\hline $40-49$ & 2352 & 21,7 & 2681 & 22,2 & 2305 & 21,4 & 3495 & 21,2 & 10833 & 21,6 \\
\hline$\geq 50$ & 926 & 8,5 & 1140 & 9,4 & 937 & 8,7 & 1340 & 8,1 & 4343 & 8,7 \\
\hline Sin datos & 0 & 0,0 & 0 & 0,0 & 0 & 0 & 28 & 0,2 & 28 & 0,1 \\
\hline \multicolumn{11}{|l|}{ Postulante } \\
\hline Voluntario & 509 & 4,7 & 274 & 2,3 & 299 & 2,8 & 167 & 1 & 1249 & 2,5 \\
\hline No voluntario & 10327 & 95,3 & 11807 & 97,7 & 10461 & 97,2 & 16283 & 99 & 48878 & 97,5 \\
\hline Total tamizado & 6653 & 61,4 & 6430 & 53,2 & 5203 & 48,4 & 9977 & 60,7 & 28263 & 56,4 \\
\hline Total no tamizado & 4183 & 38,6 & 5651 & 46,8 & 5557 & 51,6 & 6473 & 39,3 & 21864 & 43,6 \\
\hline Total & 10836 & 100 & 12081 & 100 & 10760 & 100 & 16450 & 100 & 50127 & 100 \\
\hline
\end{tabular}


Tabla 2. Evolución de los marcadores para hepatitis B y hepatitis C en los donantes de sangre en el Hospital Nacional Dos de Mayo, Lima 2012-2015

\begin{tabular}{|c|c|c|c|c|c|c|c|c|c|c|}
\hline \multirow{2}{*}{ Marcador } & \multicolumn{2}{|c|}{2012} & \multicolumn{2}{|c|}{2013} & \multicolumn{2}{|c|}{2014} & \multicolumn{2}{|c|}{2015} & \multicolumn{2}{|c|}{ Total } \\
\hline & $\mathbf{n}$ & $\%$ & $\mathbf{n}$ & $\%$ & $\mathbf{n}$ & $\%$ & $\mathbf{n}$ & $\%$ & $\mathbf{n}$ & $\%$ \\
\hline Total tamizado & 6653 & 100 & 6430 & 100 & 5203 & 100 & 9977 & 100 & 28263 & 100 \\
\hline \multicolumn{11}{|l|}{$\mathrm{HBsAg}$} \\
\hline Reactivo & 20 & 0,3 & 38 & 0,6 & 31 & 0,6 & 67 & 0,7 & 156 & 0,6 \\
\hline No reactivo & 6633 & 99,7 & 6392 & 99,4 & 5172 & 99,4 & 9910 & 99,3 & 28107 & 99,4 \\
\hline \multicolumn{11}{|l|}{ Anti-HBc } \\
\hline Reactivo & 139 & 2,1 & 317 & 4,9 & 283 & 5,4 & 726 & 7,3 & 1465 & 5,2 \\
\hline No reactivo & 6514 & 97,9 & 6113 & 95,1 & 4920 & 94,6 & 9251 & 92,7 & 26798 & 94,8 \\
\hline \multicolumn{11}{|l|}{ Anti-HVC } \\
\hline Reactivo & 44 & 0,7 & 50 & 0,8 & 50 & 1,0 & 88 & 0,9 & 232 & 0,8 \\
\hline No reactivo & 6609 & 99,3 & 6380 & 99,2 & 5153 & 99,0 & 9889 & 99,1 & 28031 & 99,2 \\
\hline
\end{tabular}

fue menor a lo reportado por De la Cruz et al. en el Hospital Nacional Cayetano Heredia, donde se halló una prevalencia de $0,8 \%{ }^{(8)}$; mientras que para el VHC, Pinto et al. en el referido nosocomio reportaron una proporción de positividad de $0,8 \%$ en los donantes de sangre ${ }^{(9)}$, una cifra similar a nuestro estudio. En el Hospital Nacional Docente Madre Niño San Bartolomé se reportó un promedio de frecuencia de $0,3 \%$ para $\mathrm{HBsAg}, 4,6 \%$ para HBcAg y $0,7 \%$ para VHC ${ }^{(10)}$.

La situación cambia al comparar con los hallazgos entre los donantes de un hospital de Apurímac, situado en la sierra meridional del país, donde la frecuencia de casos positivos para HBsAg y anti-HBcAg fue de 1,92 y $35 \%$ respectivamente ${ }^{(11)}$; la mayor prevalencia se vincula con el pasado de episodios de brotes de hepatitis $\mathrm{B}^{(12)}$. Vildózola et al. encontraron una asociación entre el riesgo de infección por hepatitis B y el haber nacido fuera de Lima ${ }^{(13)}$.

Según la OMS, la sangre donada por voluntarios altruistas es más segura que la sangre de donantes de reposición. Al respecto, un estudio en Mali muestra que la positividad para HBsAg fue significativamente mayor en las unidades de sangre recolectadas de los donantes de reemplazo que los donantes voluntarios $(p<0,001)$ y la positividad para VHC y HBsAg fue significativamente mayor $(p<0,001)$ en unidades de sangre recolectadas de nuevos donantes que los donantes regulares (14). En Guinea, la prevalencia de HBsAg confirmados fue significativamente menor en los donantes de reemplazo o en donantes familiares que en los donantes voluntarios por primera vez $\left(p<0,001\right.$ y $p=0,04$, respectivamente) ${ }^{(15)}$. En el valle central de California, la prevalencia de VHB fue de 0,28\% (IC95\%. 0,22 -0,33) en los donantes de primera vez y 0,01\% (IC95\%: 0,007-0,02) entre donantes repetidos ${ }^{(16)}$.

En nuestro estudio, los resultados positivos para $\mathrm{HBsAg}$ y anti-VHC se presentaron en menor proporción en los donantes no voluntarios o de reposición, dichos resultados se dan porque los donantes voluntarios, en la mayoría de los casos, vienen por primera vez, mientras que los donantes "no voluntarios" que son los donantes de reposición, han donado en más de una ocasión y se encuentran registrados en el banco de sangre. En la fase de entrevista, los hallazgos clínicos como la

Tabla 3. Reactividad para los marcadores de hepatitis $B$ y hepatitis $C$ según la categoría del donante en el Hospital Nacional Dos de Mayo, Lima 2012-2015

\begin{tabular}{|c|c|c|c|c|c|c|c|}
\hline \multirow{2}{*}{ Marcador } & \multicolumn{2}{|c|}{ No voluntario } & \multicolumn{2}{|c|}{ Voluntario } & \multicolumn{2}{|c|}{ Total } & \multirow{2}{*}{ Valor $p$} \\
\hline & $\mathbf{n}$ & $\%$ & $n$ & $\%$ & $\mathbf{n}$ & $\%$ & \\
\hline \multicolumn{8}{|l|}{ HBsAg } \\
\hline No reactivo & 27383 & 99,5 & 724 & 99,2 & 28107 & 99,4 & 0,319 \\
\hline Reactivo & 150 & 0,5 & 6 & 0,8 & 156 & 0,6 & \\
\hline \multicolumn{8}{|l|}{ Anti-HBc } \\
\hline No reactivo & 26101 & 94,8 & 697 & 95,5 & 26798 & 94,8 & 0,413 \\
\hline Reactivo & 1432 & 5,2 & 33 & 4,5 & 1465 & 5,2 & \\
\hline \multicolumn{8}{|l|}{ Anti-HVC } \\
\hline No reactivo & 27312 & 99,2 & 719 & 98,5 & 28031 & 99,2 & 0,037 \\
\hline Reactivo & 221 & 0,8 & 11 & 1,5 & 232 & 0,8 & \\
\hline Total & 27533 & 100 & 730 & 100 & 28263 & 100 & \\
\hline
\end{tabular}


Tabla 4. Reactividad para los marcadores de hepatitis $B$ y hepatitis $C$ según grupos de edad en el Hospital Nacional Dos de Mayo, Lima 2012-2015

\begin{tabular}{|c|c|c|c|c|c|c|c|c|c|c|c|c|c|}
\hline \multirow{2}{*}{ Marcador } & \multicolumn{2}{|c|}{$<20$} & \multicolumn{2}{|c|}{$20-29$} & \multicolumn{2}{|c|}{$30-39$} & \multicolumn{2}{|c|}{$40-49$} & \multicolumn{2}{|c|}{$\geq 50$} & \multicolumn{2}{|c|}{ Total } & \multirow{2}{*}{ Valor $p$} \\
\hline & $\mathbf{n}$ & $\%$ & $\mathbf{n}$ & $\%$ & $\mathbf{n}$ & $\%$ & $\mathbf{n}$ & $\%$ & $\mathbf{n}$ & $\%$ & $\mathbf{n}$ & $\%$ & \\
\hline Total & 2497 & 100 & 8872 & 100 & 8732 & 100 & 6045 & 100 & 2117 & 100 & 28263 & 100 & \\
\hline HBsAg & & & & & & & & & & & & & 0,721 \\
\hline No reactivo & 2485 & 99,5 & 8818 & 99,4 & 8684 & 99,5 & 6017 & 99,5 & 2103 & 99,3 & 28107 & 99,4 & \\
\hline Reactivo & 12 & 0,5 & 54 & 0,6 & 48 & 0,5 & 28 & 0,5 & 14 & 0,7 & 156 & 0,6 & \\
\hline Anti-HBc & & & & & & & & & & & & & $<0,001$ \\
\hline No reactivo & 2407 & 96,4 & 8510 & 95,9 & 8262 & 94,6 & 5677 & 93,9 & 1942 & 91,7 & 26798 & 94,8 & \\
\hline Reactivo & 90 & 3,6 & 362 & 4,1 & 470 & 5,4 & 368 & 6,1 & 175 & 8,3 & 1465 & 5,2 & \\
\hline Anti-HVC & & & & & & & & & & & & & 0,174 \\
\hline No reactivo & 2470 & 98,9 & 8811 & 99,3 & 8649 & 99 & 6000 & 99,3 & 2101 & 99,2 & 28031 & 99,2 & \\
\hline Reactivo & 27 & 1,1 & 61 & 0,7 & 83 & 1,0 & 45 & 0,7 & 16 & 0,8 & 232 & 0,8 & \\
\hline Donación & & & & & & & & & & & & & $<0,001$ \\
\hline No voluntario & 2405 & 96,3 & 8586 & 96,8 & 8561 & 98,0 & 5923 & 98,0 & 2058 & 97,2 & 27533 & 97,4 & \\
\hline Voluntario & 92 & 3,7 & 286 & 3,2 & 171 & 2,0 & 122 & 2,0 & 59 & 2,8 & 730 & 2,6 & \\
\hline
\end{tabular}

ictericia puede ser suficiente para no ser admitido; en los casos de ictericia puede superar el $50 \%$ la positividad para el HBsAg ${ }^{(17)}$, en nuestro país también se reportó mayor riesgo de infección por hepatitis $B$ en sujetos con antecedentes de ictericia (13). La autoexclusión voluntaria es otra alternativa para evitar el tamizaje inmunoserológico; se sabe que entre los autoexcluidos voluntarios la frecuencia de positividad para los antígenos HBs y anticuerpos contra VHC es mayor comparado con los donantes no autoexcluidos $(p<0,05)^{(18)}$.

En el presente estudio, la positividad para HBsAg y anti$\mathrm{HBc}$ se encontró en menor proporción en los menores de 50 años. Estos resultados, particularmente en el caso de los jóvenes, pueden tener relación con la incorporación de la vacuna contra la hepatitis B como parte del programa de inmunización en la década de los 90. La vacuna contra la hepatitis $B$ se ha traducido en reducción importante en la prevalencia de HBsAg ${ }^{(19)}$; sin embargo, el efecto no sería muy favorable en escenarios donde la cobertura de la vacuna contra HVB es deficiente ${ }^{(20)}$.

Entre las limitaciones, considerar que los resultados positivos obtenidos no fueron confirmados, puesto que la función del banco de sangre es hacer el tamizaje, mientras que la confirmación y seguimiento de los resultados le corresponde al Programa de Control de Enfermedades de Transmisión Sexual y SIDA-PROCETSS.

En conclusión, en el periodo 2012 a 2015 entre los donantes de sangre, el $0,6 \%$ fue reactivo para $\mathrm{HBsAg}$; $5,2 \%$ para anti-HBc y $0,8 \%$ para Anti-VHC. La reactividad positiva para HBsAg y anti-VHC fueron en mayor proporción en los donantes voluntarios, la reactividad para HBsAg y anti-HBc fueron en mayor proporción en las personas de 50 a más años de edad. Los donantes voluntarios fueron en mayor proporción en los sujetos menores de 20 años.

Agradecimientos: a los colaboradores y equipo de soporte técnico del banco de sangre del Hospital Nacional Dos de Mayo.

Contribución de los autores: JMQ y JFR participaron en la concepción, diseño del estudio, análisis e interpretación de datos y redacción del artículo. CDS participó en la concepción del estudio, aporte de material de estudio, asesoría administrativa y aprobación de la versión final. HMS participó en asesoría técnica y aprobación de la versión final.

Fuentes de financiamiento: autofinanciado

Conflictos de interés: los autores declaran no tener conflictos de interés.

\section{REFERENCIAS BIBLIOGRÁFICAS}

1. Organización Mundial de la Salud. Estrategia Mundial del Sector de la Salud contra las Hepatitis Víricas para 2016-2021 [Internet]. 2015. Disponible en: http://www.who.int/ reproductivehealth/STI_strategy_ abridged_es.pdf
2. Organización Mundial de la Salud. Prevención y control de las hepatitis virales: Marco para la acción mundial [Internet]. Organización Mundial de la Salud. 2012. Disponible en: http://apps.who.int/iris/ bitstream/10665/130014/1/WHO HSE_PED_HIP_GHP_2012.1_spa.pdf
3. Babanejad M, Izadi N, Najafi F, Alavian SM. The HBsAgPrevalence Among Blood Donors From Eastern Mediterranean and Middle Eastern Countries: A Systematic Review and Meta-Analysis. Hepat Mon. 2016;16(3):2-8. DOI: 10.5812/ hepatmon. 35664 . 
4. Cabezas C. Hepatitis viral B y delta en el Perú: Epidemiología y bases para su control. Rev Peru Med Exp Salud Publica. 2007;24(4):378-97.

5. Mayca J, Vallejos R. Hepatitis B en el Perú: Revisión 1970-2002. Rev Peru Med Exp Salud Publica. 2004;21(4):261-9.

6. Perú. Programa Nacional de Hemoterapia y Bancos de Sangre. Situación Actual.

7. Rigas AS, Sørensen CJ, Pedersen OB, Petersen MS, Thørner LW, Kotzé S, et al. Predictors of iron levels in 14,737 Danish blood donors: Results from the Danish Blood Donor Study. Transfusion [Internet]. 2014;54(March):789-96. Disponible en: https://www.ncbi.nlm. nih.gov/pubmed/24372094.

8. De la Cruz R, Barrera T, Vidal J, Rodriguez I. Marcadores serológicos de sífilis, hepatitis B y VIH en donantes de sangre en el Hospital Nacional Cayetano Heredia, Lima-Perú. Rev Med Hered. 1999;10(4):137-43.

9. Pinto J, Vidal J, Bussalleu A, Huerta J, Ramirez D, Valdivia J, et al. Infección por el Virus de la Hepatitis C en donantes del banco de sangre en el Hospital Cayetano Heredia (1998-2002). Rev GastroenterolM Peru. 2003;23(1):22-8.

10. Moya J, Julcamanyan E. Seroprevalencia de marcadores infecciosos causantes de pérdidas de hemodonaciones en el Servicio de Banco de Sangre del Hospital Nacional Docente Madre Niño San Bartolomé de enero 2008 a diciembre del 2013. Horiz Med. 2014;14(4):6-14.

11. Ramírez-Soto MC, Huichi-Atamari M. Hepatitis B en donantes de sangre de un hospital en Apurímac, Perú. Rev Peru Med Exp Salud Publica. 2012;29(1):163-4.

12. Méndez M, Arce M, Kruger H, Sánchez S. Prevalencia de marcadores serológicos de hepatitis vírica en diversos grupos de población del Perú. Bol Saint Panam. 1989;106(2):127-38.

13. Vildózola H, Bazul V, Cambillo E, Torres J, Flores ME, Ramos E. Prevalencia de la infección y factores de riesgo para Hepatitis B en dos grupos de gestantes adolescentes en relación al número de parejas sexuales. Rev Gastroenterol Peru. 2006;26:242-58.

14. Diarra A, Kouriba B, Baby M, Murphy E, Lefrere JJ. HIV, HCV, HBV and syphilis rate of positive donations among blood donations in Mali: Lower rates among volunteer blood donors. Transfus Clin Biol. 2009;16(5-6):444-7. DOI: 10.1016/j.tracli.2009.09.004

15. Loua A, Nze Nkoure G. Relative safety of first-time volunteer and replacement donors in Guinea. Transfusion. 2010;50(8):1850-1. DOI: 10.1111/j.1537-2995.2010.02718.x

16. Sheikh MY, Atla PR, Ameer A, Sadiq H, Sadle PC. Seroprevalence of hepatitis B and $\mathrm{C}$ infections among healthy volunteer blood donors in the central California valley. Gut Liver. 2013;7(1):66-73. DOI: $10.5009 /$ gnl.2013.7.1.66

17. Ochwoto M, Kimotho JH, Oyugi J, Okoth F, Kioko H, Mining S, et al. Hepatitis B infection is highly prevalent among patients presenting with jaundice in Kenya. BMC Infect Dis [Internet]. BMC Infectious Diseases; 2016;16(1):101. DOI: $10.1186 /$ s12879016-1409-2

18. Kasraian L, Tavasoli A. Positivity of HIV, hepatitis $B$ and hepatitis $C$ in patients enrolled in a confidential self-exclusion system of blood donation: A crosssectional analytical study. Sao Paulo Med J. 2010;128(6):320-3.

19. Leroi C, Adam P, Khamduang W, Kawilapat S, Ngo-Giang-Huong N, Ongwandee S, et al. Prevalence of chronic hepatitis $\mathrm{B}$ virus infection in Thailand: a systematic review and metaanalysis. Int J Infect Dis [Internet]. 2016;51:36-43. Disponible en: http:// dx.doi.org/10.1016/j.jijid.2016.08.017

20. Cabezas-Sánchez C, Trujillo-Villarroel O, Zavaleta-Cortijo C, Culqui-Lévano D, Suarez-Jara M, Cueva-Maza N, et al. Prevalencia de la infección por el virus de hepatitis B en niños menores de 5 años de comunidades indígenas de la amazonía peruana posterior a intervenciones mediante inmunización. Rev Peru Med Exp Salud Publica. 2014;31(2):204-10.

Correspondencia: Juan Morales Quispe

Dirección: Av Universitaria $N^{o}$ 5175, Los Olivos. Lima, Perú.

Teléfono: +51989521832

Correo electrónico:mdjuanmorales@gmail.com

\section{Nuestros artículos se encuentran indizados en:}

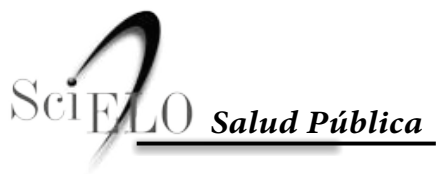

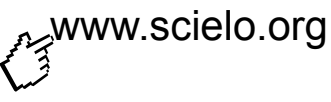

